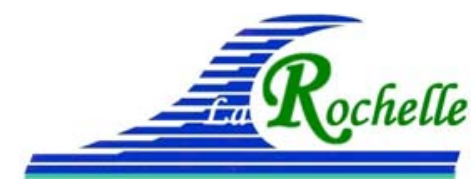

XVèmes Journées Nationales Génie Côtier - Génie Civil

La Rochelle, 29 au 31 mai 2018

DOI:10.5150/jngcgc.2018.007 ～(C) Editions Paralia CFL

disponible en ligne - http://www.paralia.fr - available online

\title{
Générateur de houle à contre-courant via un déversoir oscillant
}

\author{
Johan FOURDRINOY ${ }^{1}$, Léo-Paul EUVÉ ${ }^{1}$, \\ Jean-Marc MOUGENOT ${ }^{1}$, Germain ROUSSEAUX ${ }^{1}$
}

\author{
1. Institut Pprime, UPR 3346, CNRS-Université de Poitiers-ISAE ENSMA \\ 11 Boulevard Marie et Pierre Curie - Téléport 2, BP 30179, 86962 Futuroscope \\ Cedex, France. \\ germain.rousseaux@univ-poitiers.fr
}

\section{Résumé :}

Nous présentons un nouveau type de batteur à houle générant des ondes à contrecourant imaginé à l'Institut Pprime de Poitiers sur la base d'une idée de Jean-Marc Mougenot. Ce générateur de vagues est composé d'un double seuil: le premier permettant de réguler la hauteur d'eau moyenne, le second, muni d'un moteur linéaire, de générer les ondes. Nous utilisons un canal hydraulique équipé de sondes acoustiques permettant des mesures locales et non-intrusives de la hauteur d'eau. Dans ce travail, nous explorons différentes configurations afin de vérifier la linéarité des ondes sinusoïdales générées. Nous regardons l'effet du courant sur les ondes générées en faisant varier le débit et la hauteur d'eau asymptotique, et nous traçons des fonctions de transfert présentant le rapport entre l'amplitude de la houle et l'amplitude de déplacement mécanique, en fonction de la fréquence du batteur.

Mots-clés : Hydrodynamique, Batteur à houle, Fonction de transfert, Interaction houlecourant.

\section{Introduction}

Dans la nature, la propagation de la houle peut avoir lieu en présence de courant (PEREGRINE, 1976 ; JONSSON, 1990 ; THOMAS \& KLOPMAN, 1997 ; MAISSA et al., 2013). Il est donc utile de pouvoir reproduire ces conditions en laboratoire. Il existe de nombreux types de générateurs de houle mécanique en canal hydrodynamique (batteur piston, volet, pneumatique, à coin...) (PATEL, 2013), dont le fonctionnement est basé sur l'oscillation d'une condition limite (SCLAVOUNOS, 2002). Ces batteurs sont installés sur de nombreux canaux et sont sources de beaucoup d'études (DAOUD \& KOBUS, 1993 ; SCHÄFFER, 1996). Une étude à l'Ecole Centrale de Nantes propose un dispositif générant à la fois houle et courant (BABARIT et al., 2016). Lors d'une expérience à l'Institut Pprime il fut remarqué que l'oscillation manuelle verticale du seuil en fin de canal avait pour effet d'envoyer à contre-courant des vagues. L'ajout d'un moteur linéaire sur un second seuil permit de produire des vagues sinusoïdales se propageant dans le canal. 


\section{Thème 1 - Hydrodynamique côtière}

Ce travail a pour but de vérifier la qualité de l'onde générée, en s'assurant qu'elle n'est pas accompagnée d'effets non-linéaires qui compliqueraient la compréhension physique $\mathrm{du}$ fonctionnement du batteur. Nous choisissons donc de diminuer l'amplitude des vagues afin d'échapper à une cascade d'effets non-linéaires, aggravés par la présence de courant : création d'harmoniques liés, libres (EUVE \& ROUSSEAUX, 2016), instabilité de type Benjamin-Feir (BENJAMIN \& FEIR, 1967; SHUGAN \& al., 2014), déferlement. Nous souhaitons relier l'amplitude de la houle au déplacement mécanique du batteur. En particulier, nous cherchons à tracer une fonction de transfert présentant l'amplitude des vagues par rapport au déplacement mécanique, en fonction de la fréquence du batteur. Nous cherchons à comprendre l'effet de la variation des paramètres de l'écoulement (débit et hauteur d'eau asymptotique), sur cette fonction de transfert.

\section{Présentation du batteur et de la méthode de mesure}

Le batteur est formé d'un double seuil (figure 1). Le premier seuil est réglable à la main à l'aide d'une manivelle avec un pas de $2 \mathrm{~mm}$, et permet de venir jouer sur le niveau d'eau moyen comme un déversoir classique (NOUGARO 1967). Un second seuil, fixé sur le premier, génère des ondes à contre-courant. Sa position est commandée par l'intermédiaire d'un moteur linéaire PS01-23x160H-HP-R de la marque LinMot, et d'un boitier de commande. Le moteur offre un déplacement compris entre $-3 \mathrm{~cm}$ et $+3 \mathrm{~cm}$. Le logiciel LinMot de TransTechnik permet un échange utilisateur / moteur linéaire. Le seuil est taillé en biseau et agit comme pour un déversoir. La chute d'eau entraîne les ondes à co-courant, elles ne peuvent donc se réfléchir à l'extrémité du canal et par la suite venir interférer avec les autres ondes en remontant à contre-courant le canal.
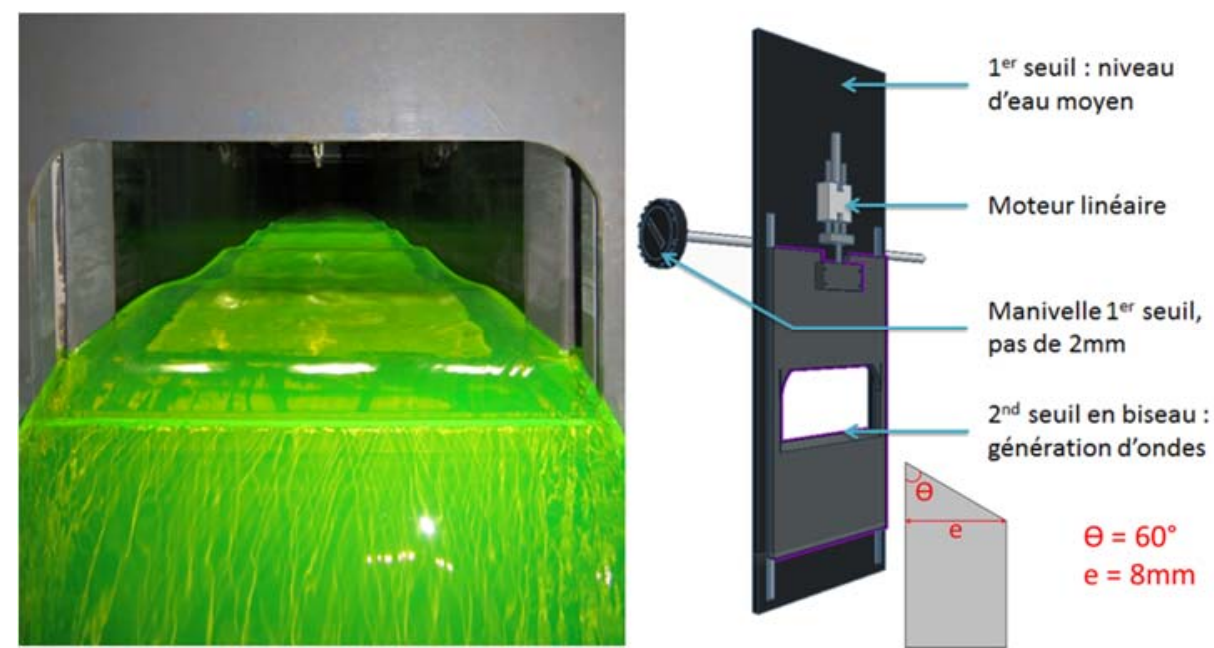

Figure 1. (GAUCHE) Photo de la région du seuil avec une houle centimétrique générée. (DROITE) Schéma du batteur à houle installé à l'Institut Pprime (http://www.univpoitiers.fr/recherche/plateformes-technologiques/hydrodynamique-environnementale-phe-/). 


\section{XVèmes Journées Nationales Génie Côtier - Génie Civil \\ La Rochelle, 29 au 31 mai 2018}

En négligeant la tension de surface ainsi que l'impact de l'amplitude des vagues sur la hauteur d'eau, en s'assurant alors d'avoir une cambrure faible (EUVÉ et al., 2016) $k a \ll 1$, les ondes générées suivent la relation de dispersion 2D linéarisée des ondes de gravité issue de la théorie linéaire des vagues en profondeur d'eau constante (PEREGRINE, 1976 ; MAISSA et al., 2013 ; FALTOT et al., 2014):

$k=\sqrt{k_{x}^{2}+\left(\frac{n \pi}{W}\right)^{2}} \quad \omega=U k_{x} \pm \sqrt{g k \tanh (k h)} \quad(U \leq 0)$

Avec $k_{x}$ le nombre d'onde longitudinal, et $n$ un entier naturel représentant l'ordre du mode transverse pouvant apparaître, $W$ la largeur du canal, $U$ la vitesse du fluide supposée constante et uniforme sur la verticale et la largeur du canal, et $\omega$ la pulsation de l'onde.

Ce batteur est installé à l'extrémité d'un canal de dimension $\mathrm{L}=680 \mathrm{~cm} \times \mathrm{W}=39 \mathrm{~cm} \times$ $\mathrm{H}=60 \mathrm{~cm}$, alimenté par une pompe $\mathrm{PCM}$ à rotor excentré et à palier "Moineau" avec une technologie de vis sans fin à débit constant. Un convergent, deux grilles et un nid d'abeille en début de canal permettent d'imposer un profil de vitesse "bouchon". Nous mesurons les hauteurs dans le canal à l'aide de sondes acoustiques Microsonic mic $+340 /$ IU/TC permettant une mesure ponctuelle non intrusive d'une précision au dixième de millimètre avec une fréquence d'acquisition de $200 \mathrm{~Hz}$. Ces sondes sont placées au centre du canal dans le sens de sa largeur.

Le canal possédant une longueur finie, une onde incidente se propage le long du canal et se réfléchit à son extrémité. Nous observons alors une interférence entre les ondes incidente et réfléchie qui créée une enveloppe modulant l'amplitude des vagues le long du canal. Afin d'isoler l'onde incidente, nous travaillons donc sur le "temps de vol", défini comme l'intervalle de temps entre le passage de l'onde incidente sous le capteur placé au début du canal (à $2 \mathrm{~m}$ du batteur) et l'arrivée de l'onde réfléchie sous ce même capteur. La méthode de mesure (figure 2) consiste donc à démarrer simultanément le batteur et l'enregistrement de la longueur d'onde, et à arrêter l'enregistrement lorsque l'interférence est visualisée par les capteurs, ce qui se traduit par une variation de l'amplitude.

Cette méthode ne permet cependant pas de mesurer proprement les ondes incidentes de faibles fréquences. Pour ces fréquences, une approximation du temps de vol est $\tau \approx$ $2 L c /\left(c^{2}-U^{2}\right)$ (avec $L$ la longueur séparant les sondes acoustiques de l'extrémité du canal et $c=\sqrt{g h}$ en supposant $k h \ll 1)$, dans le cas d'ondes longues linéaires avec $k a \ll 1$ : ainsi le nombre de périodes possiblement mesurées est :

$N=\tau f \underset{f \rightarrow 0}{\longrightarrow} 0$ (les calculs détaillés sont dans le rapport de FOURDRINOY, 2017). 


\section{Thème 1 - Hydrodynamique côtière}

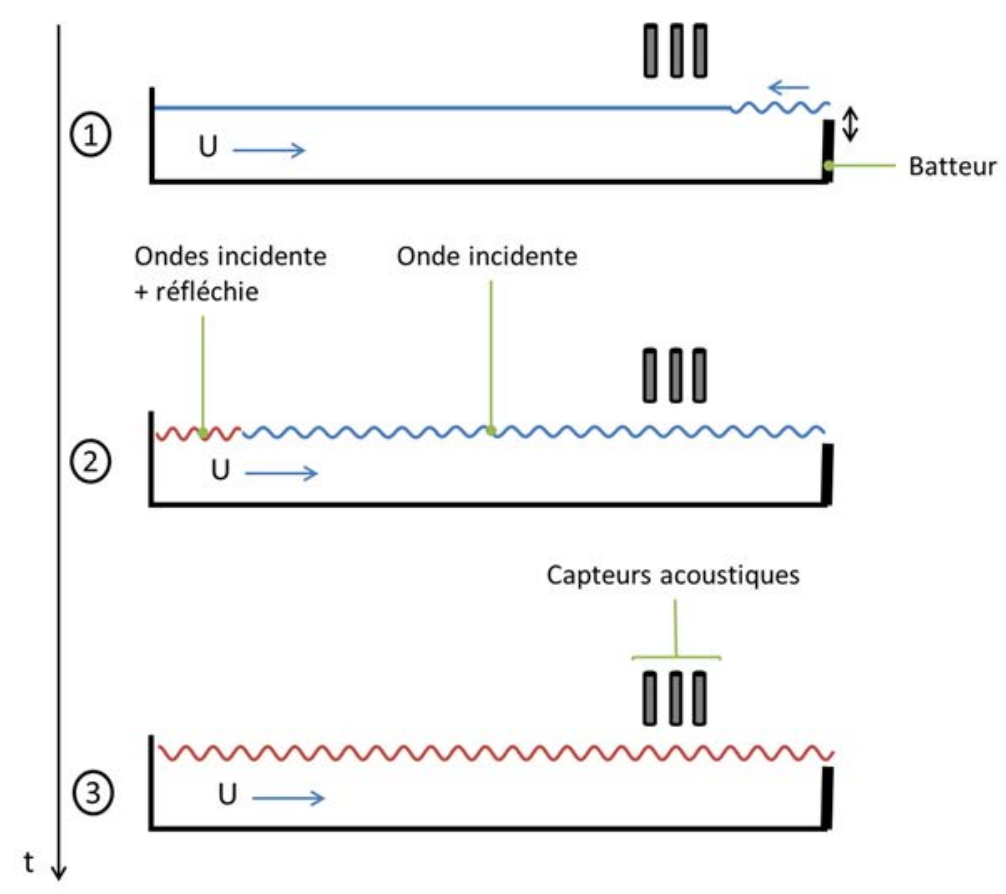

Figure 2. Méthode de mesure de l'amplitude des ondes incidentes. (1) : Démarrages de l'enregistrement de la hauteur d'eau par les sondes acoustiques et du batteur en parallèle. (2) : L'onde incidente se propage dans le canal et se réfléchit à son extrémité. (3) : L'interférence entre ondes incidente et réfléchie est totalement établie dans le canal. Seul l'enregistrement de l'étape 2 permet d'extraire l'amplitude de l'onde incidente.

\section{Résultats}

\subsection{Première mesure d'une fonction de transfert}

Nous nous plaçons à un débit de $2.5 \mathrm{~L} / \mathrm{s}$, avec une hauteur d'eau dynamique de $11.8 \mathrm{~cm}$ via le réglage du premier seuil. Cela nous donne une vitesse bouchon $U=Q / W h=$ $0.054 \mathrm{~m} / \mathrm{s}$ et un nombre de Froude dans le canal $\mathrm{Fr}=U / \sqrt{g h}=0.049<1$ : les ondes se propagent. Nous envoyons comme consigne au batteur un déplacement sinusoïdal d'amplitude mécanique $z_{m}=3 \mathrm{~mm}$ avec une fréquence variant entre $0.5 \mathrm{~Hz}$ et $4 \mathrm{~Hz}$. Trois sondes sont placées à $2.00 \mathrm{~m}, 2.22 \mathrm{~m}$ et $2.44 \mathrm{~m}$ du batteur et enregistrent à ces positions la hauteur d'eau avec la méthode du temps de vol (figure 2). A l'aide de transformées de Fourier de ces mesures, on peut lire l'amplitude de la houle sur l'amplitude de la fréquence fondamentale. On normalise ensuite l'amplitude de la houle par l'amplitude mécanique de l'oscillation du seuil.

Sur les courbes obtenues pour chaque position (figure 3), on remarque qu'elles se superposent aux plus faibles fréquences $(f \leq 2 \mathrm{~Hz})$, mais sont disparates pour les plus hautes. Ces écarts semblent apparaitre avec les ondes transversales correspondant aux modes pairs. En effet, le mode transverse d'ordre $n=2$ apparaît à la pulsation $\omega_{2}$ établi pour $k_{x}=0$ : 


\section{XVèmes Journées Nationales Génie Côtier - Génie Civil \\ La Rochelle, 29 au 31 mai 2018}

$$
\omega_{2}=\sqrt{g k_{y} \tanh \left(k_{y} h\right)}=\sqrt{g \frac{2 \pi}{W} \tanh \left(\frac{2 \pi}{W} h\right)}=12.3 \mathrm{rad} / \mathrm{s},
$$

ce qui équivaut à une fréquence $f_{2}=1.96 \mathrm{~Hz}$. Pour continuer l'exploration de la fonction de transfert sur toute la plage de fréquences, nous moyennons les résultats des 3 capteurs. Nous affichons par des barres verticales l'écart-type de ces mesures (figure 3). Nous notons que nous ne distinguons pas de particularité à la fréquence d'apparition des ondes transverses du mode 1 , ici $f_{1}=1.21 \mathrm{~Hz}$.
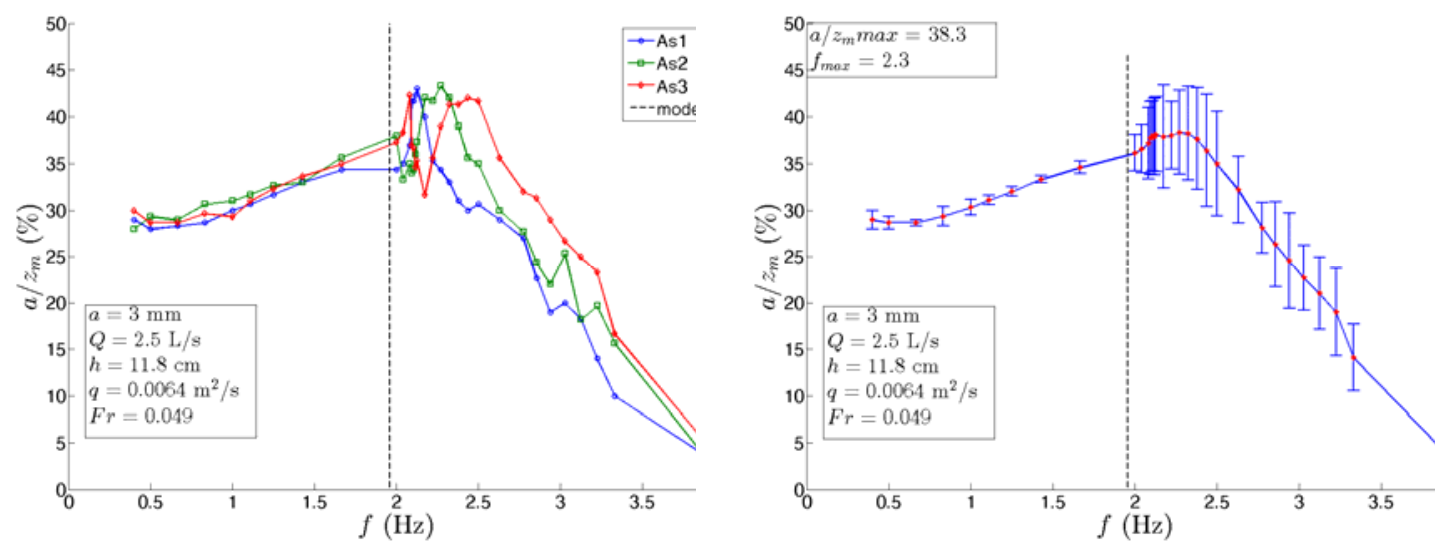

Figure 3. (GAUCHE) Tracés du rapport entre l'amplitude des ondes et le déplacement mécanique en fonction de la fréquence, selon 3 positions : courbe bleue à $2 m d u$ batteur, courbe verte à $2 m 22$, et courbe rouge à $2 m 44$. La droite verticale en pointillés noirs représente la fréquence d'apparition du mode transverse d'ordre 2. (DROITE) Fonction de transfert moyennée sur les trois courbes précédentes. Les barres verticales représentent l'écart-type entre les 3 mesures.

\subsection{Evolutions de la fonction de transfert selon le régime}

Nous réalisons par une méthode identique le tracé de la fonction de transfert dans des régimes différents, en faisant varier le débit ou la hauteur d'eau asymptotique. Sept fonctions de transfert ont été mesurées pour les paramètres du tableau 1.

Ces régimes permettent de comparer des cas conservant un paramètre constant (la hauteur d'eau asymptotique, le débit, la vitesse ou le nombre de Froude).

Tableau 1. Paramètres associés aux fonctions de transfert.

\begin{tabular}{llllllll}
\hline Régime & $\mathbf{1}$ & $\mathbf{2}$ & $\mathbf{3}$ & $\mathbf{4}$ & $\mathbf{5}$ & $\mathbf{6}$ & $\mathbf{7}$ \\
\hline $\boldsymbol{Q}(\mathbf{L} / \mathbf{s})$ & 3.9 & 2.9 & 2.5 & 3.9 & 3.9 & 10 & 4.0 \\
$\boldsymbol{H}(\mathbf{c m})$ & 20.0 & 15.0 & 11.8 & 14.9 & 8.1 & 15.0 & 6.2 \\
$\boldsymbol{U}(\mathbf{m} / \mathbf{s})$ & 0.05 & 0.05 & 0.054 & 0.067 & 0.123 & 0.171 & 0.165 \\
$\boldsymbol{F r}$ & 0.036 & 0.041 & 0.05 & 0.055 & 0.138 & 0.141 & 0.212 \\
\hline
\end{tabular}




\section{Thème 1 - Hydrodynamique côtière}

Sur la figure 4 (gauche) on remarque dans un premier temps qu'une variation du débit ou de la hauteur d'eau entraîne une modification de la fonction de transfert. Cependant, on note toutefois que ces fonctions suivent une même tendance (figure 4, droite) : une valeur asymptotique pour les faibles fréquences, suivie d'une pente $p_{1}$ croissante. Une chute de pente $p_{2}$ fait tendre l'amplitude de la houle générée vers zéro. La chute particulière de la courbe du régime 7 (tracé jaune sur la figure 4 à gauche) est due à un problème lors de la mesure : les capteurs acoustiques ne peuvent mesurer les hauteurs de la houle si la cambrure des vagues devient trop importante. Or, dans ce régime, les amplitudes des vagues dépassent les $3 \mathrm{~mm}$, ce qui implique des cambrures supérieur à $k a \geq 0.11$, les capteurs n'arrivent alors plus à mesurer.
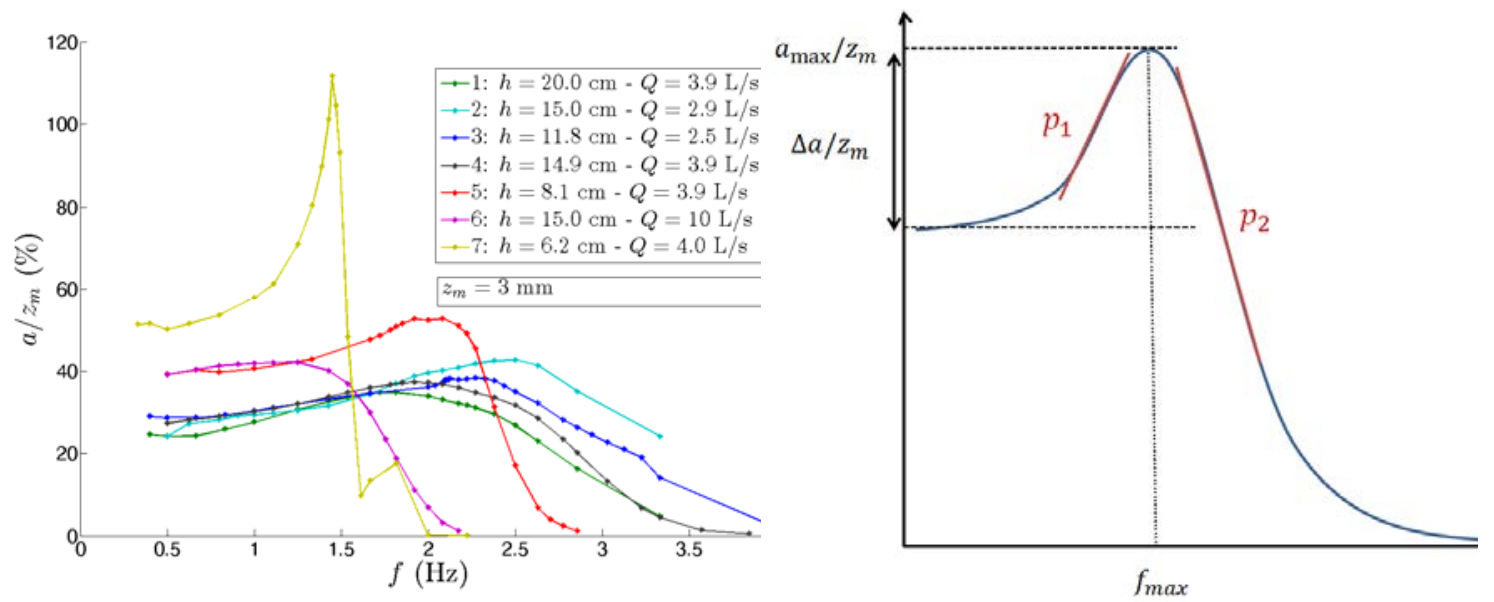

Figure 4. (GAUCHE) Fonctions de transfert dans sept régimes différents.

(DROITE) Forme supposée de la fonction de transfert en fonction de la fréquence.

La valeur asymptotique de la fonction de transfert à basses fréquences semble pilotée par le nombre de Froude. Si le nombre de Froude augmente alors l'amplitude de la houle à faibles fréquences augmente. Pour des régimes où la hauteur d'eau asymptotique et le débit diffèrent, mais où le nombre de Froude est similaire (par exemple les régimes 5 et 6), la fonction de transfert tend, à basses fréquences, vers une valeur identique. Cette tendance rappelle celle d'un filtre passe-bas. Il est donc naturel de tracer les fonctions de transfert en décibel en fonction d'une fréquence normalisée par une fréquence de coupure comme pour un diagramme de Bode. Le décibel a pour définition $X_{\mathrm{dB}}=10 \log \left(P_{1} / P_{2}\right)$ avec $P$ la puissance, or pour une vague d'Airy l'énergie vaut $\left(\rho g a^{2}\right) / 2$. Lorsque nous passons en décibel, nous avons donc $X_{\mathrm{dB}}=20 \log \left(a / z_{m}\right)$. Les fréquences de coupure sont déterminées de façon empirique. La figure ${ }^{10_{5}}$ met en évidence la tendance commune des fonctions de transfert tracées pour chaque régime. Cette représentation permet de mettre en évidence un effet similaire à une résonnance pour les régimes à faibles hauteurs d'eau asymptotiques. 


\section{XVèmes Journées Nationales Génie Côtier - Génie Civil \\ La Rochelle, 29 au 31 mai 2018}

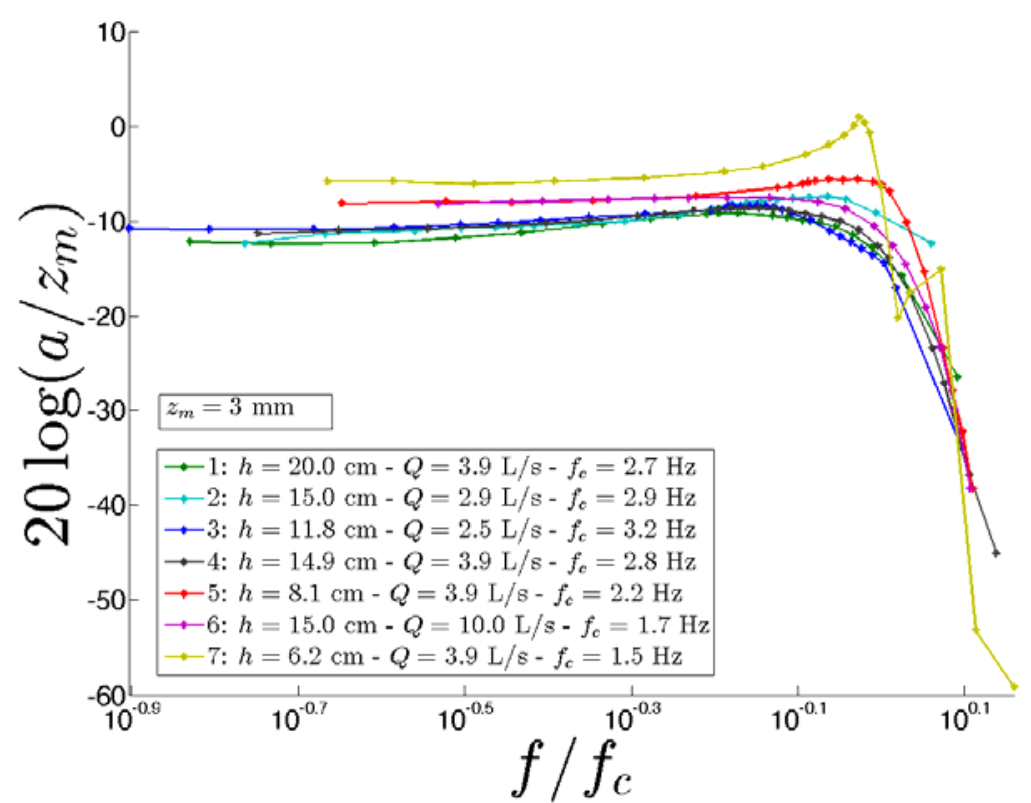

Figure 5. Fonctions de transfert en décibel en fonction de la fréquence adimensionnée par une fréquence de coupure déterminée empiriquement.

Pour comprendre la raison de la chute des amplitudes pour les plus hautes fréquences, et dans la continuité de notre analogie avec un filtre passe-bas, nous nous sommes intéressés au déphasage entre le déplacement mécanique et le déplacement de la veine du déversoir au-dessus du seuil en bout de canal. Pour cela, nous mesurons à l'aide de deux sondes acoustiques, déclenchées en parallèle, la hauteur d'eau au-dessus du seuil et la position de ce seuil. En comparant ces deux mesures, nous pouvons facilement lire un déphasage entre les deux signaux. Nous nous plaçons dans le premier régime testé ( $h=11.8 \mathrm{~cm}, Q=2.5 \mathrm{~L} / \mathrm{s}$ ) et obtenons les déphasages montrés au tableau 2 en passant dans l'espace de Fourier.

Pour les fréquences trop élevées, l'hydraulique ne parvient pas à suivre l'oscillation de la condition limite. Ceci induit un déphasage qui est donc une cause de la chute en amplitude.

Tableau 2 Déphasages obtenus en passant dans l'espace de Fourier.

\begin{tabular}{llllll}
\hline$f(\mathrm{~Hz})$ & 0.64 & 1.28 & 3.2 & 3.84 & 6.4 \\
\hline$f / f_{c}\left(f_{c}=3.2 \mathrm{~Hz}\right)$ & 0.2 & 0.4 & 1 & 1.2 & 2 \\
\hline$\phi\left({ }^{\circ}\right)$ & -3.8 & 4.5 & -22.5 & -34.3 & -54.6 \\
\hline
\end{tabular}

\subsection{Linéarité des ondes générées}

Nous nous assurons de la linéarité des ondes générées (figure 6) en étudiant la transformée de Fourier de la houle (figure 7). Nous n'observons qu'un très léger pic au 


\section{Thème 1 - Hydrodynamique côtière}

second harmonique ( $\leq 10 \%$ de l'amplitude de la fréquence fondamentale) pour les amplitudes les plus fortes, c'est-à-dire pour des faibles hauteurs d'eau ou de forts débits, et des fréquences de l'ordre de $1.5 \mathrm{~Hz}$. Il s'agit d'un harmonique lié (EUVE \& ROUSSEAUX, 2016). La génération d'harmonique est donc extrêmement faible ce qui peut se justifier par des cambrures faibles (cf. le seuil en cambrure $k a_{\text {seuil }} \approx 0.2$, mis en évidence dans notre précédente étude rapportée dans EUVÉ et al., 2016). Le pic observé à $0.4 \mathrm{~Hz}$ sur la figure 7 à gauche provient de la pompe. A faible débit il a été remarqué que la pompe génère une perturbation à cette fréquence. En pratique, le niveau d'eau moyen oscille dans son ensemble.
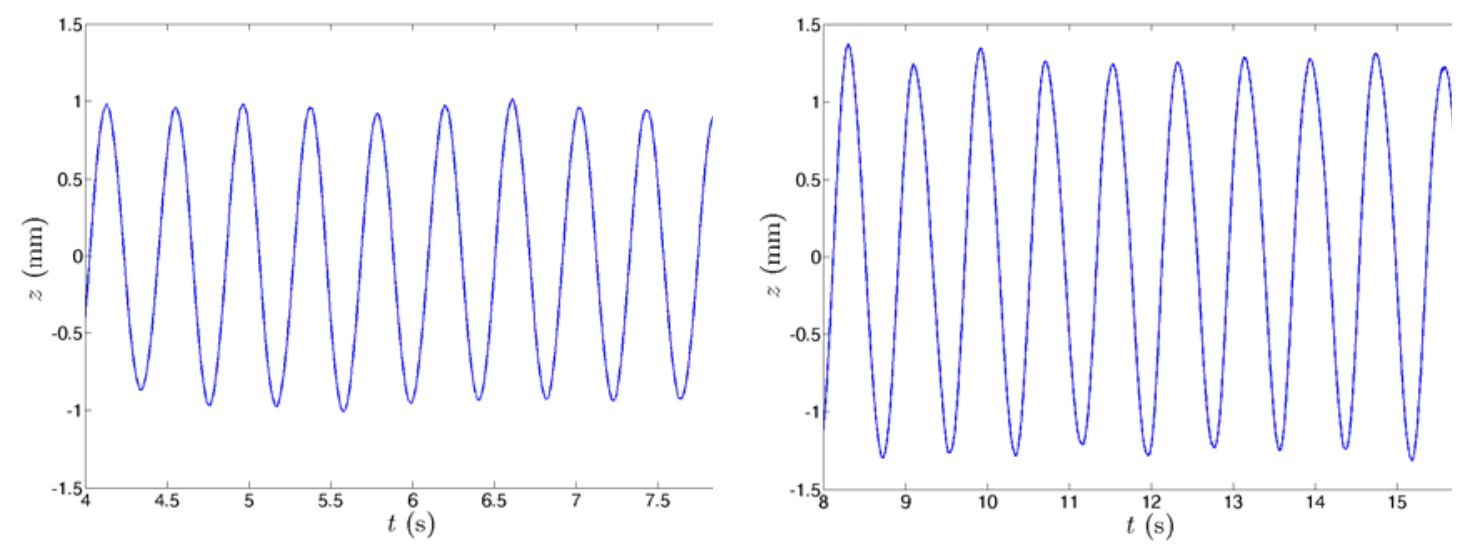

Figure 6. Variation de la hauteur d'eau dans le temps à $2 m$ du batteur. (GAUCHE) dans le cas du régime 1 pour $f=2.44 \mathrm{~Hz}$. (DROITE) dans le cas du régime 5 pour $f=1.25 \mathrm{~Hz}$. Fréquence d'acquisition $200 \mathrm{~Hz}$, lissage de la courbe par un algorithme de Savitzky-Golay (avec un polynôme de degré 3 et une largeur de 20 points).
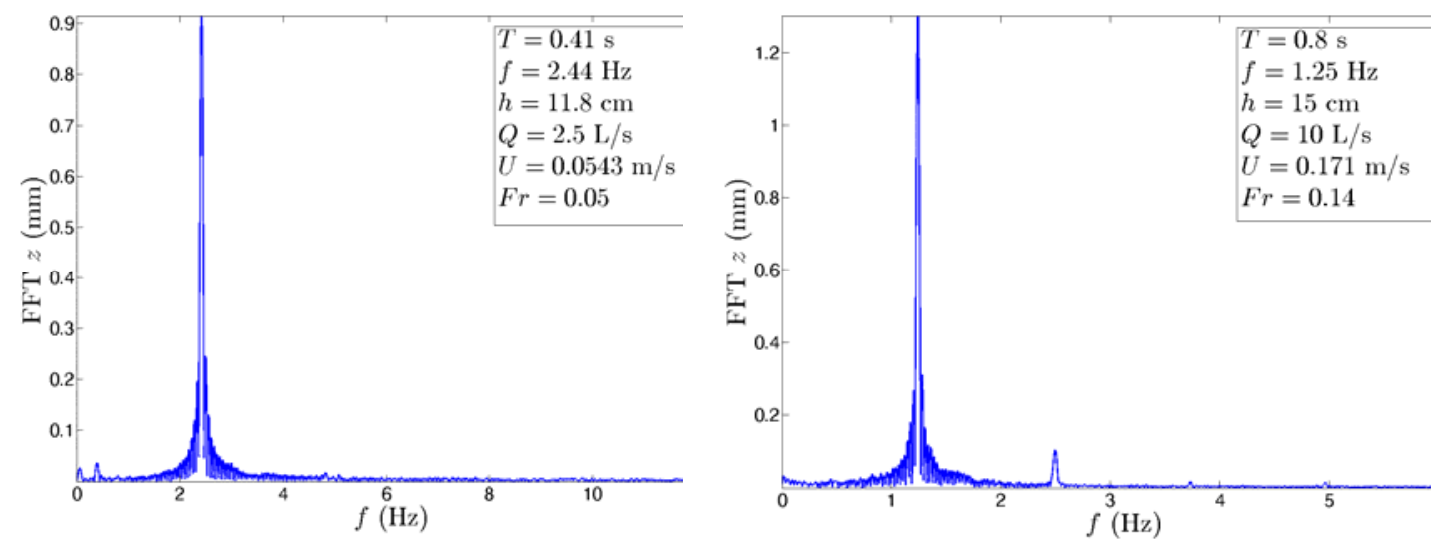

Figure 7. Transformées de Fourier de l'onde incidente : (GAUCHE) dans le cas du régime 1 pour $f=2.44 \mathrm{~Hz}$. L'amplitude du $1^{\text {er }}$ harmonique vaut $2 \%$ de celle du fondamental. (DROITE) dans le cas du régime 5 pour $f=1.25 \mathrm{~Hz}$. L'amplitude du $1^{\text {er }}$ harmonique vaut $8 \%$ de celle du fondamental. 


\section{XVèmes Journées Nationales Génie Côtier - Génie Civil \\ La Rochelle, 29 au 31 mai 2018}

\section{Conclusion}

L'étude des vagues à contre-courant trouve des applications dans divers domaines comme les spectres de houles (KROGSTAD \& TRULSEN, 2010) ou les bloqueurs pneumatiques (BEYNET \& BOUGIS, 2008), et nécessite des outils adéquats. Ce nouveau batteur à contre-courant par oscillation d'un déversoir permet de générer des ondes de très faibles amplitudes accompagnées de peu d'harmoniques. La complexité du modèle qui présente de nombreux paramètres, aussi bien géométriques (canal, forme du biseau), qu'induits par le courant (vitesse, hauteur d'eau statique), ne permet pas d'obtenir une fonction de transfert unique. Malgré ce constat, nous avons mis en avant une tendance commune qui rappelle celle d'un filtre passe-bas, ce qui peut être une première approche de la compréhension physique du batteur. Une mesure par vélocimétrie par image de particules au niveau du déversoir offrirait une compréhension accrue de l'écoulement et des indices sur l'impact du débit et de la hauteur d'eau asymptotique sur la génération d'onde. De même, la mise en place d'une étude numérique (CFD) au niveau de ce seuil pourrait poursuivre le sujet et permettre d'optimiser le générateur en jouant sur la géométrie du biseau. La linéarité des ondes millimétriques générées offre la possibilité de réaliser des études autour de l'interaction onde-courant en s'affranchissant de nombreuses complexifications. Suite à cette étape et à une meilleure compréhension de la physique, nous pourrons alors si on le souhaite, générer des ondes centimétriques non linéaires (le batteur pouvant atteindre une gamme d'amplitude de six centimètres crêtes à creux).

\section{Remerciements}

Les auteurs souhaitent remercier vivement les organismes suivants pour leur soutien et les subventions accordées: la Mission Interdisciplinaire du CNRS (PEPS PTI 2014 DEMRATNOS); les Universités de Poitiers et de Tours à travers l'ACI Interaction Houle-Courant de l'Université de Poitiers 2013 et l'ARC Poitiers-Tours 2014 ; l'ANR à travers le programme HARALAB (ANR-15-CE30-0017-04) ainsi que le FEDER 35790-2012 qui a financé le canal. Les auteurs remercient Laurent Dupuis et Ludovic Mascarenhas pour leur aide technique dans le montage mécanique du batteur.

\section{Références bibliographiques}

BABARIT A., ARNAL V., DELVOYE S., WACKERS J., DAVOUST L., BONNEFOY F. (2016). Numerical and experimental study of a wave and current generation system using axial-flow pumps. XV ${ }^{\text {èmes }}$ Journées de l'Hydrodynamique.

BARBE, A., BOYER, P., COULOMB, R., DE SAINT-MARTIN, J. M., NOUGARO, J. (1967). Loi hauteur-débit d'un déversoir en mince paroi fonction de la charge totale $H$ et valable dans une large plage d'utilisation. La Houille Blanche, $\mathrm{n}^{\circ} 3$, pp 249-256. https://doi.org/10.1051/lhb/1967016 


\section{Thème 1 - Hydrodynamique côtière}

BENJAMIN T.B., FEIR J.E. (1967). Instability of periodic wavetrains in nonlinear dispersive systems. Journal of Fluid Mechanics, Vol. 27, pp 417-430. https://doi.org/10.1098/rspa.1967.0123

BEYNET J. M., BOUGIS J. (2008). Processus de développement d'un débarcadère auto protégé contre la houle par un brise-lames pneumatique. $\mathrm{X}^{\mathrm{èmes}}$ Journées Nationales Génie Côtier Génie Civil, pp 687-698. https://doi.org/10.5150/ingcgc.2008.065-B

DAOUD B., KOBUS J.-M. (1993). Génération et mesure de la houle aléatoire en

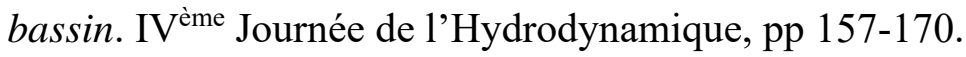

EUVÉ L.-P., ROUSSEAUX G. (2016). Génération non-linéaire d'harmoniques après une conversion linéaire en interaction houle-courant. XIV ${ }^{\mathrm{èmes}}$ Journées Génie Côtier Génie Civil, pp 29-38. https://doi.org/10.5150/jngcgc.2016.004

FALTOT P.-J., BELLANGER R., MOUGENOT J.-M., ROUSSEAUX G. (2014). Interaction Houle-Courant: Mesure de la Relation de Dispersion. XIII ${ }^{\text {èmes }}$ Journées Génie Côtier Génie Civil, pp 79-92. https://doi.org/10.5150/jngcgc.2014.010

FOURDRINOY J. (2017). Qualification d'un batteur à houle par mesure de déformée de surface libre. Rapport de stage N7 encadré par ROUSSEAUX G. Sept. 2016 - Févr. 2017.

JONSSON I.G. (1990). Wave-current interactions. In The Sea (ed. B. Le Mehaute and D. M. Hanes), pp 65-120, John Wiley.

KROGSTAD H.E., TRULSEN K. (2010), Interpretations and observations of ocean wave spectra, Ocean Dynamics, Vol. 60, pp 973-991. https://doi.org/10.1007/s10236-010-0293-3 MAISSA P., ROUSSEAUX G., STEPANYANTS Y. (2013). Influence of shear-flow vorticity on wave-current interaction. 7th Internat. Conference on Coastal Dynamics, Arcachon, pp 1137-1146. URL : http://www.coastaldynamics2013.fr/pdf_files/103_Maissa_Philippe.pdf PATEL M. H. (2013). Dynamics of offshore structures. Butterworth-Heinemann. Chap 9: Model testing of offshore structure.

PEREGRINE D.H. (1976). Interaction of water waves and currents. Adv. Appl. Mech., Vol. 16, pp 9-117. https://doi.org/10.1016/S0065-2156(08)70087-5

SCHÄFFER H.A. (1996). Second-order wavemaker theory for irregular waves. Ocean Engineering, Vol. 23(1), pp 47-88. https://doi.org/10.1016/0029-8018(95)00013-B

SCLAVOUNOS P. (2002). Ocean Wave Interaction with Ships and Offshore Energy Systems (13.022). Massachusetts Institute of Technology MIT.

SHUGAN I., SAPRYKINA Y., KUZNETSOV S., YANG R. (2014). Frequency downshifting of wave spectra and formation of freak waves on non-uniform opposing current. Coastal Engineering Proceedings, Vol. 1(34), waves.24. https://doi.org/10.9753/icce.v34.waves.24

THOMAS G.P., KLOPMAN G. (1997). Wave-current interactions in the nearshore region. In Gravity Waves in Water of Finite Depth. J. N. Hunt, Editor, Advances in Fluid Mechanics, Vol. 10, CMP, Southampton. 